\title{
Effect of Needle Tip Position on Contrast Media Dispersion Pattern in Transforaminal Epidural Injection Using Kambin's Triangle Approach
}

This article was published in the following Dove Press journal: Journal of Pain Research

\author{
Jongseok Lee' \\ Daehyun Jo ${ }^{2}$ \\ Shinmi Song' \\ Dahee Park (D) ${ }^{\prime}$ \\ Dohyeong Kim (D) \\ Jinyoung $\mathrm{Oh}\left(\mathbb{D}^{\prime}\right.$
}

'Department of Anesthesiology and Pain Medicine, Gangnam Severance Hospital, Yonsei University College of Medicine, Seoul, Republic of Korea; ${ }^{2}$ Pain Center, Pohang Wooridul Hospital, Pohang-si, Gyeongsangbuk-do, Republic of Korea
Correspondence: Jinyoung Oh

Department of Anesthesiology and Pain Medicine, Gangnam Severance Hospital, Yonsei University College of Medicine,

2II Eonju-Ro, Gangnan-Gu, Seoul,

Republic of Korea

Tel +82-2-2019-3600

$\mathrm{Fax}+82-2-2019-4833$

Email sgcms3@gmail.com
Purpose: Dispersion of contrast media into the anterior epidural space is correlated with better outcomes after transforaminal epidural injection (TFEI). Needle tip position is an important factor affecting the pattern of contrast media dispersion. It is difficult to advance the needle medially to the interpedicle line with a conventional approach, especially in a severe spinal stenosis. But, with Kambin's triangle approach, the needle can be advanced more medially even in the severe stenosis. We aimed to compare contrast media dispersion patterns according to the needle tip position in TFEI with Kambin's triangle approach.

Patients and Methods: This single-center retrospective study analyzed fluoroscopic data of patients who underwent TFEI from March 2019 to July 2019. Data on the history of lumbar spinal fusion surgery and MRI findings were collected. The needle tip position was evaluated in three positions on fluoroscopic images (final anteroposterior [AP] view): extraforaminal (EF), lateral foramen (LF), and medial foramen (MF). Contrast media dispersion into the epidural space (epidural pattern) in the AP view was evaluated as a dependent variable. The relationship between the contrast media dispersion pattern and needle tip position was analyzed, and other factors affecting the contrast media dispersion pattern were identified.

Results: Ninety-eight TFEI cases were analyzed (51 LF, $35 \mathrm{MF}$, and $12 \mathrm{EF}$ ). An epidural pattern of dispersion was observed more frequently in the LF and MF groups than in the EF group. The LF and MF groups showed no significant difference in epidural pattern frequency. On logistic regression analysis, needle tip position emerged as a major factor influencing epidural pattern, while other factors including spine conditions had no significant effect.

Conclusion: Positioning the needle tip medial to the pedicle helps in the spread of the contrast media into the epidural space during TFEI with Kambin's approach. Factors other than the needle tip position did not significantly affect the contrast media dispersion pattern. Keywords: fluoroscopy, spinal stenosis, disc herniation, epidural space, needle tip, contrast media

\section{Introduction}

Transforaminal epidural injection (TFEI) is an effective modality in managing lumbosacral radicular pain. ${ }^{1,2}$ It has the advantage of delivering medications, including local anesthetics or steroids, to areas closest to pain generators in the anterior epidural space; hence, it is more efficient in relieving pain than the caudal or interlaminar approach. ${ }^{3}$ Since the drugs can be injected directly around the nerve root through the intervertebral foramen, this method can be used as either diagnostic or therapeutic selective nerve blocks. 
Several studies about TFEI have focused on selectivity for diagnostic purpose. Lateral needle tip position and low volume of drug injection are known to be factors for good selectivity of the injection. ${ }^{4-6}$ However, there is limited evidence regarding the diagnostic value of TFEI. ${ }^{3,7}$ On the contrary, the therapeutic efficacy of TFEI is supported by clinical evidence and guidelines. The American Society of Interventional Pain Physicians suggested that the evidence of TFEI is strong for short-term and moderate for longterm improvement in managing lumbar radiculopathy. ${ }^{7}$ Appropriate TFEI may reduce the rate of surgical interventions in patients with lumbar radiculopathy. ${ }^{8}$ This efficacy is a result of the direct dispersion of drugs into the anterior epidural space where structures that cause radiculopathies, such as dorsal root ganglion or compressed spinal nerves are located. ${ }^{3,9,10}$ The greatest advantage of TFEI is that it can directly inject the drug at the pathologic site. ${ }^{6}$ In the conventional approach, which uses a safe triangle technique, the needle tip is placed below the underside of the pedicle and above the exiting spinal nerve root, within the half of the neural foramen. With this approach, the medication can be injected close to the nerve root, and the risk of damaging the dura mater is decreased. However, given the potential for damaging the anterior spinal artery and spinal nerve root, it is sometimes difficult to insert the needle into the anterior epidural space properly. ${ }^{11}$ Moreover, in many cases of severe foraminal stenosis, the needle is unable to reach the epidural space after passing the intervertebral foramen. ${ }^{3,12}$

Kambin's triangle approach can overcome the aforementioned issues. Kambin's triangle is defined as a right triangle over the dorsolateral disc. The hypotenuse is the exiting nerve root, the base is the superior border of the caudal vertebra, and the height is the dura/traversing nerve root. ${ }^{13}$ The needle can be inserted into the epidural space with less nerve or arterial damage, and the drug can spread better into the epidural space where the pathologies are located. ${ }^{3,14}$ However, the anatomical position of superior articular process can make this approach more difficult to the needle tip inside, and, Kambin's triangle approach has more risk of intradiscal injection than conventional approach. ${ }^{15}$ These challenges make it difficult to properly position the needle tip in the epidural space with Kambin's triangle approach.

Several studies have investigated the correlations between contrast media dispersion patterns and clinical outcomes in TFEI. ${ }^{10,13,14,16-18}$ Contrast media dispersion into the anterior epidural space is related to a better outcomes. ${ }^{10,16,17}$ Moreover, it can be affected by the final needle tip position before injection. Desai et $\mathrm{al}^{19}$ found that the final needle tip position in lateral view in TFEI is associated with the contrast media flow pattern and with clinical outcome of patients.

Some physicians argue that in severe lumbar spinal stenosis, the contrast media may not spread into the anterior epidural space even if the needle is in the proper position. With the conventional approach (safe triangle technique), it is recommended to advance the needle not medial to the 6 o'clock position of the pedicle to avoid dural sleeve injury or intrathecal injection. ${ }^{20}$ The contrast media would not disperse into the epidural space at this needle tip position when the lumbar spinal stenosis is severe. However, with Kambin's triangle approach, the needle can be advanced more medially avoiding the nerve root or artery in the route. In this study, we aimed to compare the contrast media dispersion pattern according to the needle position in TFEI with Kambin's triangle approach. Our hypothesis was that as the needle advances more medially, the contrast media disperses more into the epidural space. We conducted this study to define the proper transforaminal epidural injection for back pain with or without radiating leg pain from degenerative lumbar spine including lumbar disc herniation or spinal stenosis.

\section{Patients and Methods}

This retrospective study using medical records from a single institution was approved by the institutional review board of a Gangnam Severance hospital (3-20190166). The requirement for patient informed consent for participation was waived because of the retrospective study design and the utilization of anonymized data.

Fluoroscopic images collected during the procedure and magnetic resonance imaging (MRI) scans of patients who underwent TFEI for the treatment of low back and/or radiating leg pain due to lumbar disc herniation or spinal stenosis were collected from March 2019 to July 2019. Patients who received TFEI according to the protocol (described later: TFEI protocol) for radiating leg pain with or without back pain caused by lumbar spinal stenosis or lumbar disc herniation were included in this study. If the procedure was performed at more than two sites at once, each site was considered a separate case. Patients whose MRI findings obtained within 6 months of the procedure or fluoroscopic images obtained during the procedure were not available in their medical records, those 
who underwent the procedure with a protocol other than the TFEI protocol, or patients who showed abnormal contrast media dispersion patterns (intradiscal, intravascular, subdural, intrathecal) during the procedure were excluded from the analysis. We also collected patient age, sex, history of lumbar spinal fusion surgery, and lumbar MRI findings which include the degree of foraminal stenosis, lateral recess stenosis, and disc herniation at the site of the procedure performed.

\section{TFEI Protocol}

TFEI was performed for patients who complained of low back and/or radiating leg pain from lumbar disc herniation or spinal stenosis. The level of TFEI was determined based on the dermatome of the patient's pain and lumbar MRI findings. The procedure was performed under fluoroscopic guidance by a skilled practitioner with more than 3 years of experience. On the fluoroscopy table, the patient was placed in a prone position with a pillow under the pelvis. First, the fluoroscopic beam was adjusted so that the spinous process was in the center and the vertebral endplates of the target level were aligned in a straight line in the anteroposterior (AP) view. It was then rotated ipsilaterally to align the superior articular process of the vertebra below to the 6 o'clock position of the pedicle above (oblique view).

A 20 G Tuohy needle with a bent tip was used. The needle entry point was defined as the point slightly lateral to the superior articular process of the caudal vertebra of the target level in the oblique view. The area surrounding the entry point was disinfected with hexetidine, followed by skin infiltration with $1 \%$ lidocaine. The needle was then inserted through Kambin's triangle using the tunnel view technique, along the axis of the fluoroscopic beam. After the orientation of the needle was determined, the fluoroscopic beam was rotated to the AP view, and the needle was advanced to the lateral margin of the pedicle in the AP view. The fluoroscope beam was then rotated to the lateral view, and the needle was advanced to pass beyond the front half of the vertebral foramen (Figure 1). Next, the fluoroscope beam was rotated to the AP view again, and the location of the needle tip was confirmed, followed by the injection of $1 \mathrm{~mL}$ of contrast media (Bonorex $300 \mathrm{INJ}$, Central Medical Service, Seoul, Korea). Immediately after the injection of contrast media, a fluoroscopic image was taken to confirm that there was no abnormal pattern (such as intravascular, intradiscal, or intrathecal pattern), and then the drug was injected.

\section{Needle Tip Position}

The needle tip position was evaluated in three positions in the final AP view of TFEI. It was defined as "extraforamen (EF)" when the final needle tip was located lateral to the lateral margin of pedicle, "lateral foramen (LF)" when the needle tip was located between the lateral margin and half of the pedicle (lateral to the interpedicular line, respectively), and "medial foramen (MF)" when the needle tip was positioned at the medial side of the half of the pedicle (medial to the interpedicular line) in AP view (Figure 2).

\section{Contrast Media Dispersion Pattern}

The contrast media dispersion pattern was evaluated in the final AP view after a total of $1 \mathrm{~mL}$ of contrast media was injected. If the contrast media spread only along the nerve root but not into the epidural space, the dispersion pattern was defined as the nerve root pattern, and if the contrast media spread into the epidural space, the dispersion pattern was defined as the epidural pattern (Figure 3). If the pattern was abnormal (intradiscal, intravascular, subdural, intrathecal), the needle was repositioned. The prepared drug was injected after the nerve root or epidural pattern was confirmed.

\section{Data Collection}

Patient age, sex, and history of lumbar spinal fusion surgery were collected as demographic data. The severity of foraminal stenosis, lateral recess stenosis, and disc herniation was evaluated on MRI at the site of the TFEI. Foraminal stenosis grade was assessed according to the method of Lee et $\mathrm{al}^{21}$ in the sagittal view at the site of the TFEI: grade 0 , no obliteration of the perineural fat and nerve root; grade 1, perineural fat obliteration surrounding the nerve root in the transverse or vertical direction; grade 2, perineural fat obliteration surrounding the nerve root in the vertical and transverse directions; and grade 3, nerve root collapse. Lateral recess stenosis grade was assessed according to the method of Splettstosser et $\mathrm{al}^{22}$ in the transverse view at the level of the TFEI: grade 0 , normal; grade 1, lateral recess stenosis without deviation of the nerve root; grade 2, deviation of the nerve root; and grade 3, compressed nerve root. Disc herniation was classified as normal, protruded, or extruded according to the latest recommendations of the combined task forces of the North American Spine Society, American Society of Spine Radiology, and American Society of Neuroradiology. ${ }^{23}$ 

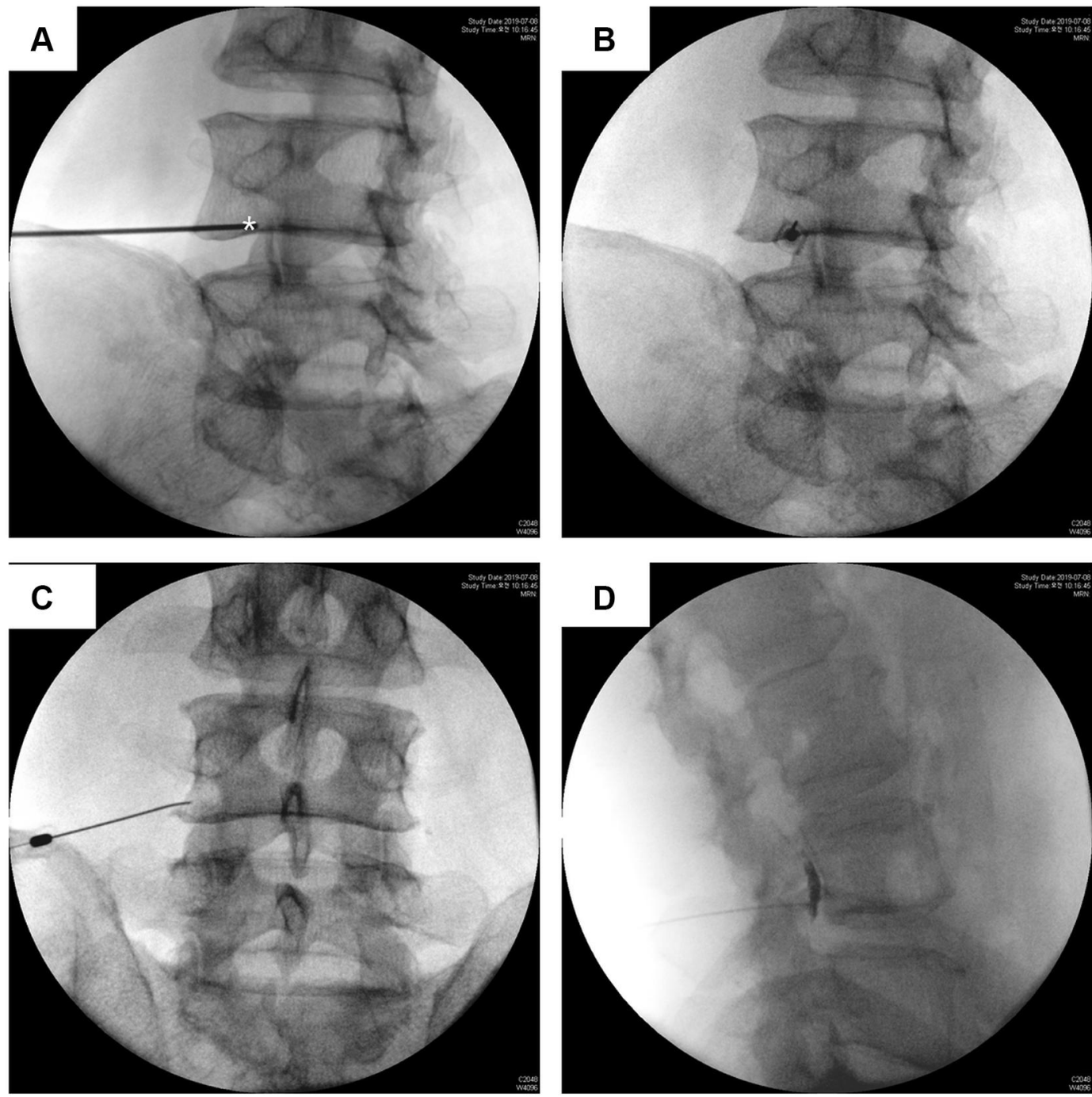

Figure I Fluoroscopic images of selective epidural transforaminal epidural injection (STE). (A) skin entry point was just lateral to ipsilateral superior articular process of the lower vertebrae of the target level (asterisk) on oblique view. (B) The needle was inserted with tunnel view technique until it can be orientated. (C) The needle was advanced to the lateral margin of the pedicle in the AP view. (D) The needle was advanced to pass beyond the front half of the vertebral foramen and anterior epidural space was confirmed by contrast media.

\section{Analysis}

Statistical analysis was performed using R 3.6.0 (R Foundation for Statistical Computing, Vienna, Austria). Categorical variables were presented as numbers and frequencies. The relationship between the contrast media pattern and needle tip position was analyzed by a chisquare test or Fisher's exact test with Bonferroni correction. ${ }^{24}$ We also performed a logistic regression analysis for finding whether other factors affected the contrast media pattern.

\section{Results}

Of the 107 patients who received TFEI for their low back and/or radiating leg pain from lumbar disc herniation or spinal stenosis, 91 had records of MRI performed within 6 months of the procedure. Of these patients, 76 underwent TFEI at a single level, and 15 underwent TFEI at two levels. Of the 106 cases, eight showed abnormal patterns and were excluded from the analysis. Finally, 98 cases (male $=51$; female $=47$ ) of TFEI were analyzed (Figure 4). Patients were $66.1 \pm 13.4$ years old, and 22 cases had a history of lumbar spinal fusion surgery.

The procedure was most often performed at L45, followed by L5S1, and predominantly on the right side (Table 1). There were 51 cases of LF, 35 of MF, and 12 of tip EF. No significant differences were found in lumbar surgery spinal fusion history, level of the procedure, and MRI findings according to needle tip position (Table 2). 


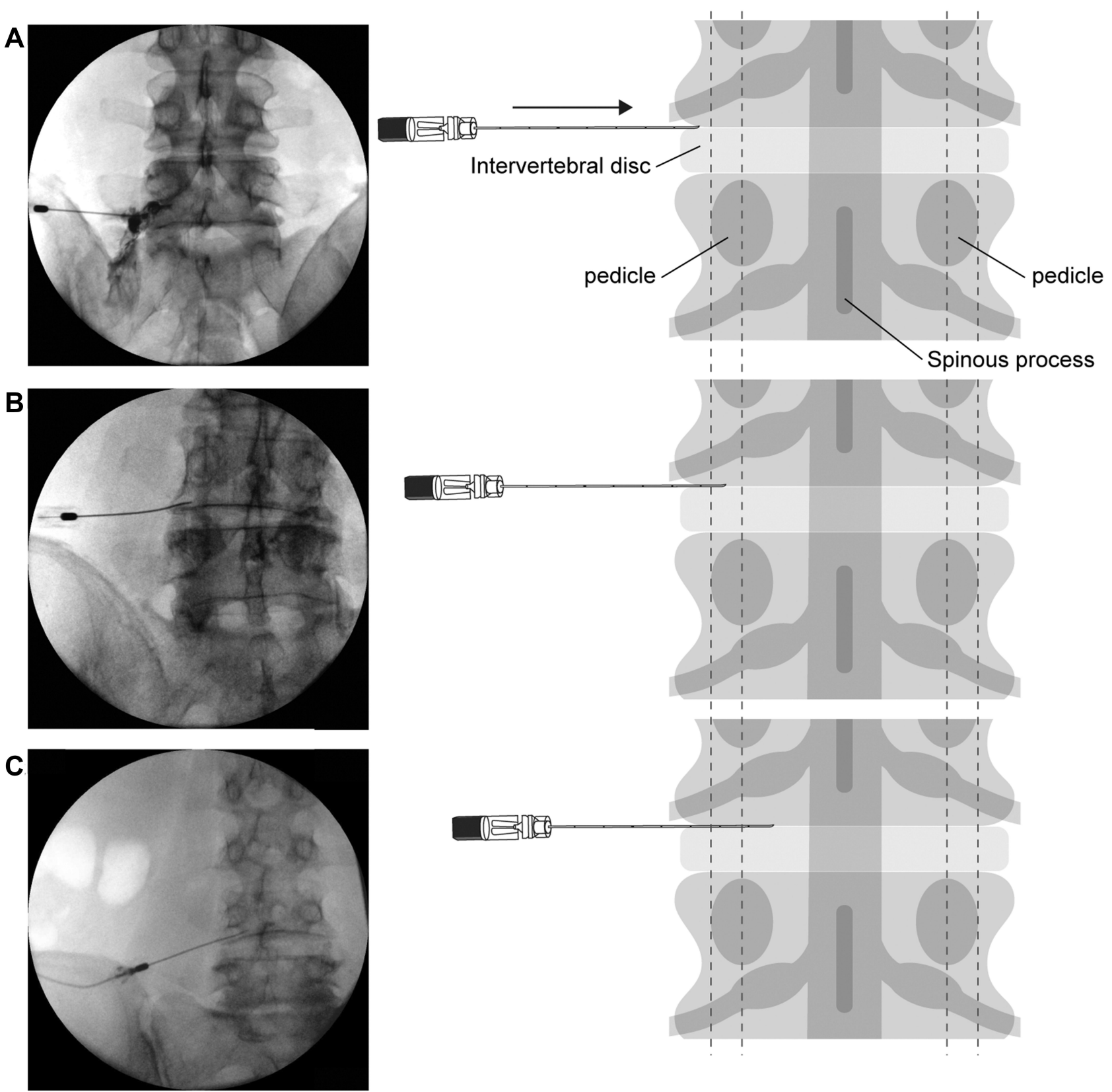

Figure 2 Needle tip position: (A) extraforamen, outside the pedicle; (B) lateral foramen (LF), lateral to the interpedicular line; and (C) medial foramen (MF), medial to the interpedicular line.

Epidural pattern was more frequently shown in LF and MF than in EF. In the EF group, the epidural pattern was observed in less than half (41.7\%) of the cases, while $86.2 \%$ and $97.1 \%$ of the cases involved the epidural pattern in the LF and LF groups, respectively. The differences in the frequency of epidural pattern between EF and LF and between $\mathrm{EF}$ and MF were statistically significant, while the difference between LF and MF was not (Table 3).

Logistic regression analysis was performed to identify other factors that affect the contrast media dispersion pattern. For this, we first analyzed the spreading pattern and all other factors by Student's $t$-test or Fisher's exact test. Only needle tip position showed $P$ value $<0.05$ (Supplementary material: Table S1). We then performed the logistic regression analysis, which showed that only needle tip significantly affects the spreading pattern (Table 4).

On multivariate logistic regression analyses, the needle tip position was a major factor for the epidural pattern, while the other factors were not (Table 4). Based on EF, 

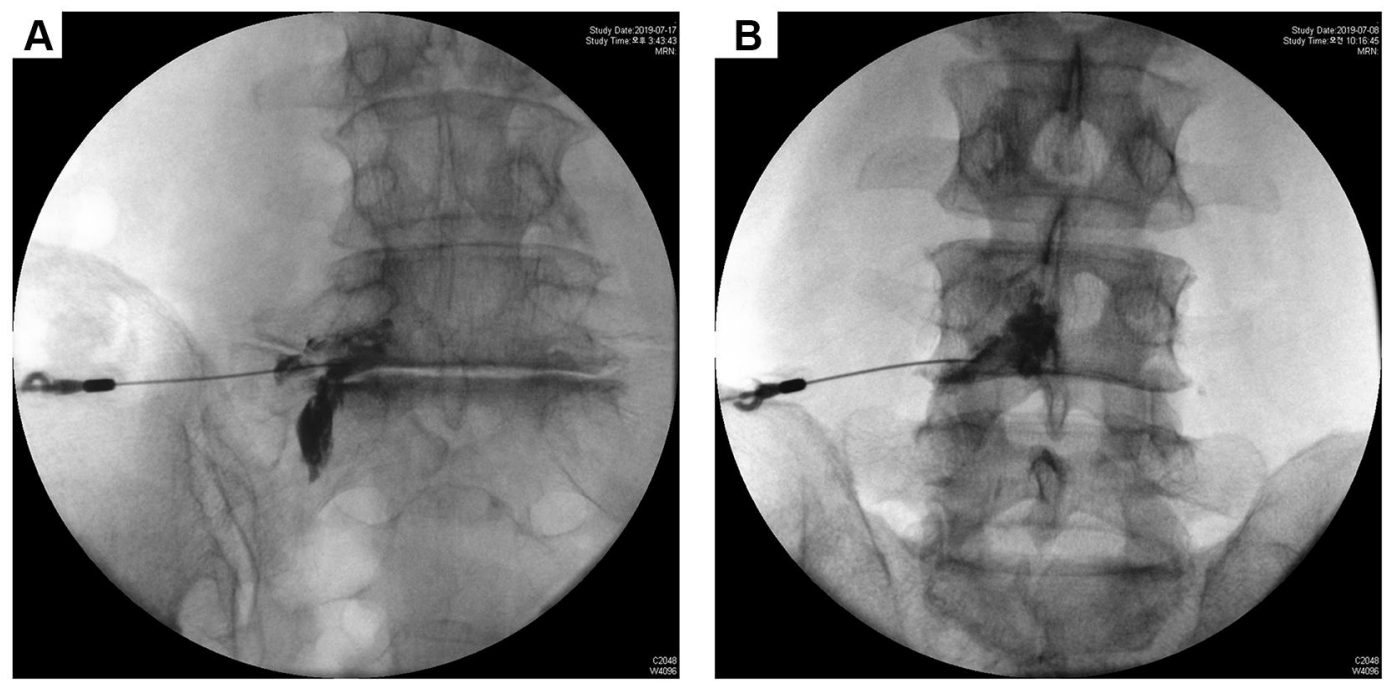

Figure 3 Contrast media pattern. (A) Nerve root: the contrast media is dispersed along the nerve root only. (B) Epidural: contrast media is dispersed into the epidural space.

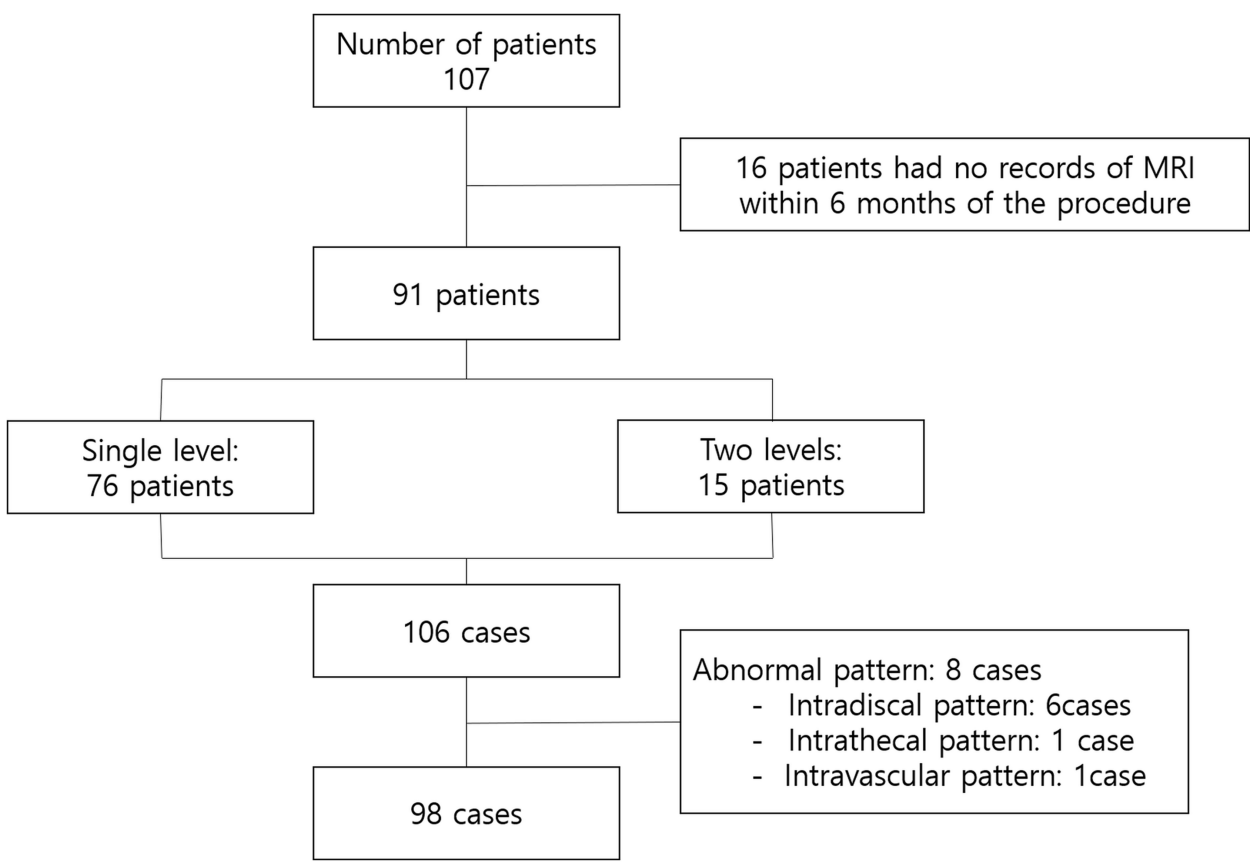

Figure 4 Flowchart of cases. Regarding abnormal patterns, two EF cases show intradiscal pattern; of the three LF cases, two show intradiscal pattern and one shows intravascular pattern; of the three MF cases, two show intradiscal pattern and one shows intrathecal pattern.

the odds ratio of LF for the epidural pattern was $3.64(95 \%$ CI 1.231-6.044) and that of MF for the epidural pattern was $5.24(95 \%$ CI $2.034-8.442)$.

\section{Discussion}

TFEI is commonly performed as treatment for leg pain radiating from the spine. Pathologically, radicular pain is caused by a compressed dorsal root ganglion or sensitized nerve roots. ${ }^{25}$ Almost these structures are located in the epidural space. That is, the target of TFEI should be the epidural space.

The primary question of this study was whether the epidural pattern appeared using a fixed amount $(1 \mathrm{~mL})$ of contrast media. Some practitioners may believe it is enough that the effect of a proper transforaminal epidural injection can be expected just by the appearance of the nerve root pattern because the drug can action on the nerve root in that condition regardless. However, it is important to properly 
Table I Sites of Selective Transforaminal Epidural Injection

\begin{tabular}{|l|l|l|l|}
\hline Level & Left & Right & Total \\
\hline L23 & 2 & 3 & 5 \\
L34 & 1 & 7 & 8 \\
L45 & 21 & 30 & 5 I \\
L5SI & 17 & 17 & 34 \\
Total & 41 & 57 & 98 \\
\hline
\end{tabular}

inject the drug into the site of pathology. For example, when we perform the TFEI for L5 radiculopathy from L4-5 intervertebral disc herniation, the Kambin's triangle TFEI would be done in the L4-5 interspace targeting the traversing L5 root which can be confirmed by epidural pattern. ${ }^{26,27}$ If only nerve root pattern of L4 root in L45 TFEI is shown, it is not appropriate injection in that case.

The amount of contrast media can be a factor that affects the contrast media dispersion pattern. The higher the amount of injected contrast media, the more likely it is that the epidural pattern will appear. We used $1 \mathrm{~mL}$ of contrast media in all cases in this study. We cannot discuss clinical outcomes like pain relief based on our results because other factors including the amount and
Table 3 Contrast Media Dispersion Patterns According to Needle Tip Position

\begin{tabular}{|l|l|l|}
\hline & Nerve Root Pattern & Epidural Pattern \\
\hline Extraforamen (EF)* & $7(58.3 \%)$ & $5(41.7 \%)$ \\
Lateral foramen (LF) & $7(13.7 \%)$ & $44(86.2 \%)$ \\
Medial foramen (MF) & I (2.9\%) & $34(97.1 \%)$ \\
\hline
\end{tabular}

Notes: Fisher's exact test showed significant differences in contrast media dispersion patterns among the three groups $(P$ value $<0.05)$. Bonferroni test was performed as a post hoc analysis. *The differences between EF and LF and between EF and MF were significant $(P$ value $<0.05)$, but the difference between $L F$ and MF was not significant $(P$ value $=0.804)$.

regimen of the injectate can affect the outcome. However, our result suggests that TFEI can be performed properly when the needle tip is in the LF or MF position.

There are concerns that the contrast media does not spread well into the epidural space in cases of severe spinal stenosis, when evaluating fluoroscopic images of TFEI. However, our data showed that the needle tip position was a factor determining the spread of drugs into the epidural space, while other factors including lumbar spinal fusion surgery history, foraminal stenosis, lateral recess stenosis, and disc herniation were not. In this study, there were 15

Table 2 Lumbar Spinal Fusion Surgery History, Level of Procedure, and MRI Findings According to Tip Position

\begin{tabular}{|c|c|c|c|c|}
\hline & Extraforamen (EF) & Lateral Foramen (LF) & Medial Foramen (MF) & $P$ value* \\
\hline Lumbar spinal fusion surgery history & & & & 0.38 \\
\hline No & 11 & 40 & 25 & \\
\hline Yes & I & 11 & 10 & \\
\hline Level & & & & 0.19 \\
\hline L23 & 0 & I & 4 & \\
\hline L34 & 0 & 3 & 5 & \\
\hline L45 & 9 & 26 & 16 & \\
\hline L5SI & 3 & 21 & 10 & \\
\hline Foraminal stenosis & & & & 0.14 \\
\hline Grade 0 & I & 15 & 16 & \\
\hline Grade I & 5 & 13 & 9 & \\
\hline Grade 2 & 2 & 15 & 5 & \\
\hline Grade 3 & 4 & 8 & 5 & \\
\hline Lateral recess stenosis & & & & 0.13 \\
\hline Grade 0 & 0 & 7 & 4 & \\
\hline Grade I & 2 & 3 & 8 & \\
\hline Grade 2 & 0 & 9 & 4 & \\
\hline Grade 3 & 10 & 32 & 19 & \\
\hline Disc herniation & & & & 0.25 \\
\hline Protrusion & 2 & 6 & 9 & \\
\hline Extrusion & 10 & 45 & 26 & \\
\hline
\end{tabular}

Note: *Tested by Fisher's exact test.

Abbreviation: MRI, magnetic resonance imaging. 
Table 4 Logistic Analysis for Factors Associated with Contrast Media Dispersion into the Epidural Space

\begin{tabular}{|l|l|l|l|}
\hline Factors & Odds Ratio & $\mathbf{9 5 \%} \mathbf{~ C l}$ & P value* \\
\hline Needle tip position* & & & \\
Extraforamen (EF) & - & - & - \\
Lateral foramen (LF) & 8.8 & $2.18-35.6$ & $<0.05$ \\
Medial foramen (MF) & 47.6 & $4.79-472.86$ & $<0.05$ \\
\hline
\end{tabular}

Note: *Compared by logistic regression analysis.

Table 5 Contrast Media Dispersion Pattern According to Needle Tip Position in Patients with Grade 3 Foraminal Stenosis and Grade 3 Lateral Recess Stenosis $(n=15)$

\begin{tabular}{|l|l|l|}
\hline & Nerve Root Pattern & Epidural Pattern \\
\hline Extraforamen (EF) & 3 & $\mathrm{I}$ \\
Lateral foramen (LF) & 0 & 6 \\
Medial foramen (MF) & $\mathrm{I}$ & 4 \\
\hline
\end{tabular}

cases of grade 3 foraminal stenosis and grade 3 lateral recess stenosis, and the contrast media dispersed into the epidural space in most cases in LF and MF, but not in EF (Table 5). That is, if the needle is placed in the appropriate position, namely, LF or MF in this study, the drug can be injected into the epidural space sufficiently, regardless of the presence of foraminal stenosis or lateral recess stenosis.

In theory, the needle advances more medially, and the possibility of complications such as dural puncture or intradiscal injection is higher. In this study, of the eight cases showing abnormal pattern, six showed intradiscal pattern, with two cases each for EF, LF, and MF. One case in MF had an intrathecal pattern, and one case in LF had an intravascular pattern. We excluded these cases from the analysis because the primary outcome of the study was the relationship between needle tip position and contrast media dispersion pattern, not the complications. The intradiscal pattern of two cases in EF suggests that it is not necessarily safe to place the needle in the lateral position, and it is important to check the state of the target of the injection by MRI before treatment (Figure 5).

Spreading pattern and abnormal pattern did not show significant difference between LF and MF in this study. And this should be further revealed by other studies. Theoretically, it is true that MF has a higher probability of complications such as intradiscal injection than LF, so this study alone can say that LF is more recommended than MF.

We used a $20 \mathrm{G}$ Tuohy needle for all cases in this study. Two types of needle can be used for TFEI: sharp tip needle (Quincke needle or Chiba needle) or blunt tip needle (Tuohy needle, Sprotte needle, or Whitacre needle). Several studies investigated the effect of needle type on intravascular injection, dura puncture risk, or nerve root damage. ${ }^{28-30}$ However, the investigated spreading pattern in our study is related to epidural pattern (or not) and it is more likely to be determined by the position of the needle tip and the state of the spine rather than the type of needle.

This study has several limitations. First, it included a small number of cases and followed a retrospective design. Second, we analyzed the contrast media dispersion pattern according to the position of the needle tip but did not analyze the relationship between differences in the pattern and actual relief from clinical symptoms; this is because the effect of the differences in the number of treatment sites and injected drugs could not be excluded. Furthermore, affected nerve in nerve root pattern and epidural pattern can be different. In the nerve root pattern, the exiting nerve is affected, but in the epidural pattern, the traversing nerve is more likely to be
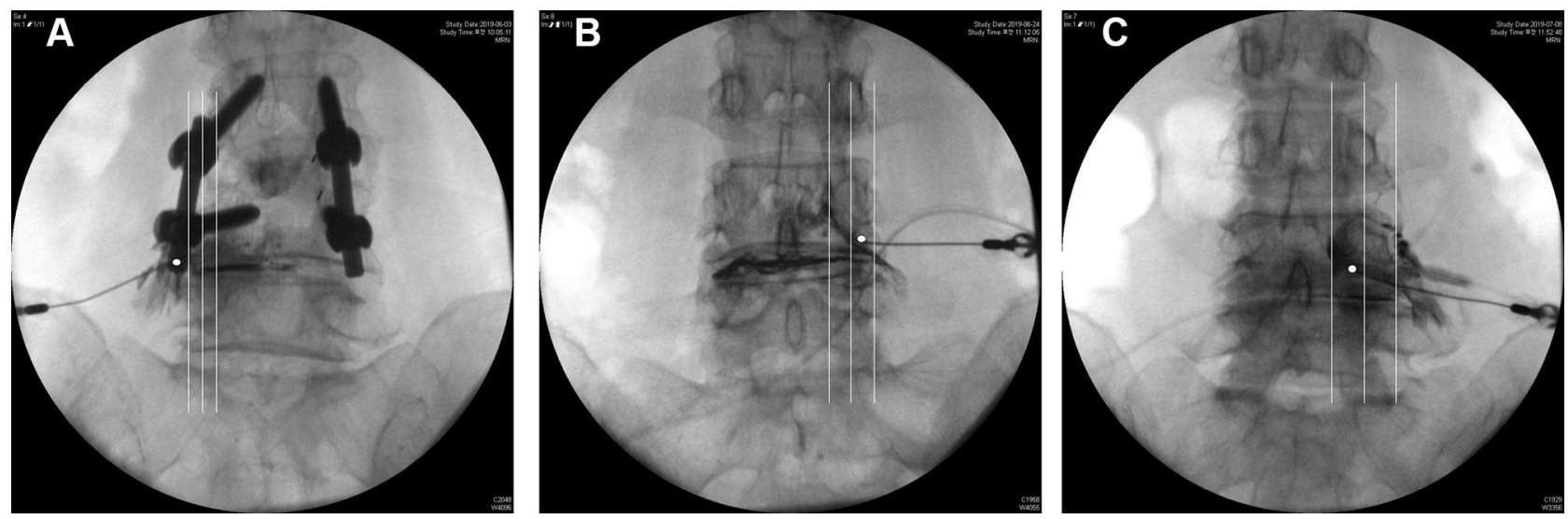

Figure $\mathbf{5}$ Intradiscal patterns (A) in extraforamen (EF) (B) in lateral foramen (LF) (C) in medial foramen (MF). White dot: position of the needle tip. White lines: medial margin, half line, lateral margin of the pedicle. 
affected. Thus, further study is needed to determine the relationship between the difference in contrast media dispersion patterns and the degree of improvement of actual symptoms. Third, in this study, we compared the spread of $1 \mathrm{~mL}$ of contrast media because injecting $1 \mathrm{~mL}$ of contrast media is our routine practice; this also helped us avoid the bias from injecting inconsistent amounts of contrast media. Of course, if the drug volume was increased, the range of drug spread also increased, which could influence the actual clinical effect. ${ }^{31}$ However, it is necessary to worry about whether the injection of even $1 \mathrm{~mL}$ of contrast media that does not spread into the epidural space can be classified as an epidural injection.

Fourth, we found no significant difference in the contrast media dispersion pattern between LF and MF. However, to clarify the difference between the two tip positions, further studies involving more cases and comparative studies on the difference between intradiscal and intrathecal injection rates are warranted. Based on this study, more than 216 samples are needed to test the difference between LF and MF with a statistical power of 0.8 under a significance level of 0.05 . Additional studies should be performed to determine differences in the position of the pedicle in relation to the midline, the relationship between the spread of contrast media and actual improvement of symptoms, and incidence of complications. Despite these limitations, our findings suggest that the needle tip position is the major factor for the epidural spread of contrast media.

\section{Conclusion}

The main factor deciding drug dispersion into the epidural space during TFEI was the needle tip position. When the needle was medial to the lateral margin of the pedicle in the AP view, the drug spreads better into the epidural space. Previous surgery and stenosis findings on MRI had no significant effect on drug dispersion. Successful TFEI can be performed by appropriately positioning the needle. Additional studies should be performed to determine the differences in the position of the pedicle in relation to the midline, the relationship between the spread of contrast medium and actual improvement of symptoms, and the incidence of complications.

\section{Abbreviations}

$\mathrm{AP}$, anteroposterior; EF, extraforamen; LF, lateral foramen; MF, medial foramen; SNRB, selective nerve root block; TFEI, transforaminal epidural injection.

\section{Data Sharing Statement}

One of the corresponding authors (sgcms3@gmail.com) will send all related data to any authority on request by an email message.

\section{Ethics Approval and Informed Consent}

The authors declare that all patients gave written informed consent before initiation of the study protocol and the study was conducted in accordance with the Declaration of Helsinki. The study was approved by the institutional review board of a tertiary care facility, Gangnam Severance Hospital (3-2019-0166). The requirement for informed consent for participation was waived because of the retrospective study design and the utilization of anonymized data.

\section{Acknowledgment}

The authors thank Medical Illustration \& Design for providing excellent support with medical illustration.

\section{Author Contributions}

All authors made a significant contribution to the work reported, whether that is in the conception, study design, execution, acquisition of data, analysis and interpretation, or in all these areas; took part in drafting, revising or critically reviewing the article; gave final approval of the version to be published; have agreed on the journal to which the article has been submitted; and agree to be accountable for all aspects of the work.

\section{Funding}

Funding information is not available.

\section{Disclosure}

The authors of this work report no conflicts of interest.

\section{References}

1. Liu J, Zhou H, Lu L, et al. The effectiveness of transforaminal versus caudal routes for epidural steroid injections in managing lumbosacral radicular pain: a systematic review and meta-analysis. Medicine. 2016;95(18):e3373. doi:10.1097/MD.0000000000003373

2. Cohen MS, Wall EJ, Brown RA, Rydevik B, Garfin SR. 1990 AcroMed award in basic science. Cauda equina anatomy. II: extrathecal nerve roots and dorsal root ganglia. Spine. 1990;15(12):12 48-1251. doi:10.1097/00007632-199012000-00003

3. Ackerman WE 3rd, Ahmad M. The efficacy of lumbar epidural steroid injections in patients with lumbar disc herniations. Anesth Analg. 2007;104(5):1217-1222, tables of contents. doi:10.1213/01.ane.00 $00260307.16555 .7 \mathrm{f}$ 
4. Irwin A, Khan AL, Fender D, Sanderson PL, Gibson MJ. The role of needle tip position on the accuracy of diagnostic selective nerve root blocks in spinal deformity. Eur Spine J. 2014;23(Suppl 1):S33-S39. doi:10.1007/s00586-014-3188-z

5. Wolff AP, Groen GJ, Wilder-Smith OH. Influence of needle position on lumbar segmental nerve root block selectivity. Reg Anesth Pain Med. 2006;31(6):523-530. doi:10.1016/j.rapm.2006.07.008

6. Furman MB, Lee TS, Mehta A, Simon JI, Cano WG. Contrast flow selectivity during transforaminal lumbosacral epidural steroid injections. Pain Physician. 2008;11(6):855-861.

7. Boswell MV, Trescot AM, Datta S, et al. Interventional techniques: evidence-based practice guidelines in the management of chronic spinal pain. Pain Physician. 2007;10(1):7-111.

8. MacVicar J, King W, Landers MH, Bogduk N. The effectiveness of lumbar transforaminal injection of steroids: a comprehensive review with systematic analysis of the published data. Pain Med. 2013;14 (1):14-28. doi:10.1111/j.1526-4637.2012.01508.x

9. Mandell JC, Czuczman GJ, Gaviola GC, Ghazikhanian V, Cho CH. The lumbar neural foramen and transforaminal epidural steroid injections: an anatomic review with key safety considerations in planning the percutaneous approach. AJR Am J Roentgenol. 2017;209(1): W26-W35. doi:10.2214/AJR.16.17471

10. Gupta R, Singh S, Kaur S, Singh K, Aujla K. Correlation between epidurographic contrast flow patterns and clinical effectiveness in chronic lumbar discogenic radicular pain treated with epidural steroid injections via different approaches. Korean J Pain. 2014;27 (4):353-359. doi:10.3344/kjp.2014.27.4.353

11. Glaser SE, Shah RV. Root cause analysis of paraplegia following transforaminal epidural steroid injections: the 'unsafe' triangle. Pain Physician. 2010;13(3):237-244.

12. Lee IS, Kim SH, Lee JW, et al. Comparison of the temporary diagnostic relief of transforaminal epidural steroid injection approaches: conventional versus posterolateral technique. AJNR Am J Neuroradiol. 2007;28(2):204-208.

13. Manchikanti L, Cash KA, Pampati V, Damron KS, McManus CD. Evaluation of lumbar transforaminal epidural injections with needle placement and contrast flow patterns: a prospective, descriptive report. Pain Physician. 2004;7(2):217-223.

14. Kim C, Moon CJ, Choi HE, Park Y. Retrodiscal approach of lumbar epidural block. Ann Rehabil Med. 2011;35(3):418-426. doi:10.5535/ arm.2011.35.3.418

15. Levi D, Horn S, Corcoran S. The incidence of intradiscal, intrathecal, and intravascular flow during the performance of retrodiscal (Infraneural) approach for lumbar transforaminal epidural steroid injections. Pain Med. 2016;17(8):1416-1422. doi:10.1093/pm/ pnv067

16. Paidin M, Hansen P, McFadden M, Kendall R. Contrast dispersal patterns as a predictor of clinical outcome with transforaminal epidural steroid injection for lumbar radiculopathy. $P M$ R. 2011;3 (11):1022-1027. doi:10.1016/j.pmrj.2011.07.020

17. Kim C, Choi HE, Kang S. Contrast spreading patterns in retrodiscal transforaminal epidural steroid injection. Ann Rehabil Med. 2012;36 (4):474-479. doi:10.5535/arm.2012.36.4.474
18. Kim WJ, Shin HY, Yoo SH, Park HS. Comparison of epidural spreading patterns and clinical outcomes of transforaminal epidural steroid injection with high-volume injectate via the subpedicular versus the retrodiscal approach. Pain Physician. 2018;21(3):269-278.

19. Desai MJ, Shah B, Sayal PK. Epidural contrast flow patterns of transforaminal epidural steroid injections stratified by commonly used final needle-tip position. Pain Med. 2011;12(6):864-870. doi:10.1111/j.1526-4637.2011.01119.x

20. Waldman SD. Atlas of Interventional Pain Management/Electronic Resource]/Wsteven D. Waldman: Elsevier/Saunders; 2015.

21. Lee S, Lee JW, Yeom JS, et al. A practical MRI grading system for lumbar foraminal stenosis. AJR Am J Roentgenol. 2010;194 (4):1095-1098. doi:10.2214/AJR.09.2772

22. Splettstosser A, Khan MF, Zimmermann B, et al. Correlation of lumbar lateral recess stenosis in magnetic resonance imaging and clinical symptoms. World J Radiol. 2017;9(5):223-229. doi:10.43 29/wjr.v9.i5.223

23. Fardon DF, Williams AL, Dohring EJ, Murtagh FR, Gabriel Rothman SL, Sze GK. Lumbar disc nomenclature: version 2.0: recommendations of the combined task forces of the North American Spine Society, the American Society of Spine Radiology and the American Society of Neuroradiology. Spine J. 2014;14 (11):2525-2545. doi:10.1016/j.spinee.2014.04.022

24. Ranstam J. Multiple P-values and Bonferroni correction. Osteoarthritis Cartilage. 2016;24(5):763-764. doi:10.1016/j.joca.20 16.01.008

25. Dower A, Davies A, Ghahreman A. Pathological basis of lumbar radicular pain: a review. World Neurosurg. 2019;128:114-121. doi:10.1016/j.wneu.2019.04.147

26. Ariffin M, Noreen F, Nor Hamdan Y, Shaharuddin AR, Azmi B. Retained intraspinal bullet: a rare case in a visitor to malaysia a case report. Malays Orthop J. 2012;6(3):45-47. doi:10.5704/ MOJ.1207.005

27. Hwang SM, Son IS, Yang PJ, Kang MS. Preganglionic epidural steroid injection through translateral recess approach. Clin Orthop Surg. 2019;11(1):131-136. doi:10.4055/cios.2019.11.1.131

28. Hernández-García JM, Reina MA, Prats-Galino A, De Andrés JA. Morphologic study of nerve root and types of needle used in transforaminal injections. Reg Anesth Pain Med. 2011;36(3):278-281. doi:10.1097/AAP.0b013e31821770d4

29. Hong J, Jung S, Chang H. Whitacre needle reduces the incidence of intravascular uptake in lumbar transforaminal epidural steroid injections. Pain Physician. 2015;18(4):325-331.

30. Smuck M, Paulus S, Patel A, Demirjian R, Ith MA, Kennedy DJ. Differential rates of inadvertent intravascular injection during lumbar transforaminal epidural injections using blunt-tip, pencil-point, and catheter-extension needles. Pain Med. 2015;16(11):2084-2089. doi:10.1111/pme.12861

31. McCarron RF, Wimpee MW, Hudkins PG, Laros GS. The inflammatory effect of nucleus pulposus. A possible element in the pathogenesis of low-back pain. Spine. 1987;12(8):760-764. doi:10.1097/ 00007632-198710000-00009
Journal of Pain Research

\section{Publish your work in this journal}

The Journal of Pain Research is an international, peer reviewed, open access, online journal that welcomes laboratory and clinical findings in the fields of pain research and the prevention and management of pain. Original research, reviews, symposium reports, hypothesis formation and commentaries are all considered for publication. The manuscript management system is completely online and includes a very quick and fair peer-review system, which is all easy to use. Visit http:// www.dovepress.com/testimonials.php to read real quotes from published authors. 